\title{
Managing urosepsis with a novel BL + BLI: A case report
}

\author{
Ashish Kr Sharma ${ }^{1}$ and Akanksha Mishra 2, * \\ ${ }^{1}$ Department of Medicine Govt Medical College, Datia. \\ ${ }^{2}$ Department of Pathology M.L.B Medical college Jhansi.
}

International Journal of Biological and Pharmaceutical Sciences Archive, 2022, 03(01), 021-023

Publication history: Received on 10 December 2021; revised on 18 January 2022; accepted on 20 January 2022

Article DOI: https://doi.org/10.53771/ijbpsa.2022.3.1.0022

\begin{abstract}
The rising resistance to Enterobacteriaceae to first-line antibiotics in treating cases of Complicated UTI (cUTI) is a serious challenge. With the rapid spread of strains producing Extended-spectrum beta-lactamases (ESBLs), few options remain in treating cases of the complicated urinary tract, especially ESBL producing E. coli, leading to urosepsis. In our case, the patient was successfully treated for ESBL, producing E. coli with Supime (cefepime+ sulbactam).
\end{abstract}

Keywords: ESBLs; cUTI; Urosepsis; Supime; Cefepime + sulbactam; E. coli

\section{Case Report}

A 67year male patient was brought to our emergency department in an unresponsive state with altered sensorium for the last two days, with continuous high-grade fever and intermittent cough for 1 month. The patient had taken treatment for fever with chills and intermittent cough from the general practitioner. The patient also reported breathlessness for the past 5 days, which increased on lying down and with coughing. However, no sputum or expectoration was reported. Past medical history was significant and included type 2 diabetes mellitus with high baseline serum creatinine equivalent to $3.4 \mathrm{mg} / \mathrm{dl}$ and hypertension for the last 12 years. The patient was on and off on medication for diabetes and hypertension. No history of urinary instrumentation or surgery in past. As the patient did not respond to the oral antibiotic treatment, he was admitted to a nursing home, and started with intravenous pip+taz for 5 days, but as the condition of the patient deteriorated, they brought him to our emergency department.

When the patient arrived at our emergency unit, the overall condition of the patient was poor with hypotension, hypothermia, tachycardia, tachypnea, leucocytosis and anaemia. Vital signs were blood pressure-72/44mmHg, pulse$118 \mathrm{bpm}$, respiratory rate- 20 breaths per min, temperature- $36.4^{\circ} \mathrm{C}$. Laboratory reports revealed a total leukocyte count of $440000 / \mathrm{mm}$, haemoglobin $11.4 \mathrm{~g} / \mathrm{dl}$ and haematocrit $32.8 \%$. Patients C-reactive protein level was deranged to $70 \mathrm{mg} / \mathrm{l}$ and pro-calcitonin level was up to $12 \mathrm{ug} / \mathrm{l}$. Based on clinical presentation and laboratory reports, the patient was diagnosed with sepsis and immediately transferred to the intensive care unit and resuscitated. Blood and urine sample was taken for routine investigations, culture and sensitivity. Chest X-ray was advised and was non-significant. Urinalysis revealed pus cells (35-40/HPF), RBC (70-80/HPF), protein (2+), blood (3+). USG of the kidney revealed decreased B/L kidney size with increased echo texture, mild ascites, and loss of cortico-medullary differentiation. A 2D echocardiography done revealed normal ejection fraction (60\%) and concentric left ventricular hypertrophy. After 48 hours, it was observed that the condition of the patient deteriorated further instead of improving with the ongoing treatment. Laboratory culture and sensitivity reports revealed MDR E. coli with resistance to pip+taz, cefoperazone sulbactam and sensitivity to cefepime + sulbactam (Supime), carbapenem, aminoglycoside and polymyxin. A provisional diagnosis of urosepsis was made. Based on c/s report, initial antibiotic therapy of was stopped, and the patient was put

\footnotetext{
${ }^{*}$ Corresponding author: Akanksha Mishra

Department of Pathology M.L.B Medical college Jhansi.

Copyright $(2022$ Author(s) retain the copyright of this article. This article is published under the terms of the Creative Commons Attribution Liscense 4.0.
} 
on cefepime + sulbactam (Supime) 3g B.I. D dose with aminoglycoside (according to renal modification doses) for 10 days along with other supportive therapy.

Patient's condition improved gradually. Repeat laboratory test revealed normalized leukocyte count with reduced CRP $(8 \mathrm{mg} / \mathrm{l})$ and pro-calcitonin levels $(<0.25 \mathrm{ug} / \mathrm{l})$. The patient was haemodynamically stable and shifted to the medicine ward for further monitoring and treatment. The patient was put on oral antibiotics and discharged on 16th-day postadmission with advice for regular follow-up.

\section{Discussion}

Urinary tract infection because of Gram-negative infections is one of the most common bacterial infection in older patients. E. coli is one of the most common uropathogen associated with community-acquired urinary tract infections, in people older than 65 years. (Linhares I). Though the spectrum of UTI may range from mild-to-moderate infections, sometimes with higher comorbidities, the illness may rise to severe sepsis. The incidence of mortality associated with sepsis, increase considerably with increasing age, with a mortality of 20-40\%. (Wagenlehner FM, Martin GS, Tal S). Multiple causes contribute to the pathogenesis of UTI in diabetic patients. Conditions like poor metabolic control of diabetes and incomplete bladder emptying because of autonomic neuropathy may all contribute to the UTI (Nitzan 0). A diabetic patient is more prone to acquiring antibiotic-resistant bacteria from the community. In our patient, the community-acquired infection with gram-negative bacteria was resistant to BL+BLI combination drugs, even though he had no significant history of hospitalization, surgery or urinary catheterization. This probably shows that there is a rise in resistant bugs in the community which could cause serious infections and not get treated by routinely used antimicrobial therapy, which was the case in our patient who had community infection but landed in urosepsis. (Venmans LMAJ, Ansari S)

The prevalence of high gram-negative pathogens is very common in the Indian subcontinent as compared to the high prevalence of gram-positive infections in the western world (Kumarasamy KK). Multiple reasons can be attributed to the rising resistance of bacteria to the first-line treatment of antimicrobials. The reasons which World Health Organisation highlights are the irrational use of antibiotics in people and animals, poor infection control, inadequate sanitary condition and inappropriate food-handling encourages the spread of antimicrobial resistance. (WHO). Thus, a rational use of antibiotic is the need of the hour so that we can spare the higher antibiotics such as the carbapenems and polymyxins for the future use. This can help in having a better hospital, community ecology and antibiotic profiles. (Trivedi M)

In our case study, the isolated pathogen was E. coli, was resistant to Pip+taz and other beta lactam+ beta-lactamases (BL+BLI) inhibitor antibiotics, but sensitive to cefepime + sulbactam+ novel AAE (Supime) and carbapenem. Moreover, the isolates which are isolated from the patients with cUTI are less likely to originate from uropathogenic strains isolated from patients with simple UTIs. Thus, the $E$. coli causing urosepsis are more likely because of the treatment failure and an important reason for the development of AMR. The early recognition of resistant bugs and treatment by using the appropriate antibiotics based on the hospital antibiogram and sensitivity pattern will help in counteracting the resistant problem and a positive outcome. We used Supime 3gm despite penems being sensitive, based on the sensitivity data and wide coverage of its activity on ESBLs and safety profile. We started getting a good clinical response by day 3 and thus continued with the treatment further till 14days. The patient recovered at the end of the therapy. This shows that Supime can be used as a better alternative against BL+BLI combination drugs like pip+taz and also preserve the carbapenem for future use.

\section{Conclusion}

E. coli is one of the prime causes of cUTI. With the increase in resistance to first-line antibiotics towards Enterobacteriaceae in treating cUTI, newer AAE therapies show a ray of hope. In our case, the patient was effectively treated with Supime and thus can be a superior treatment option than pip+taz, especially in treating serious, complicated urinary tract infection.

\section{Compliance with ethical standards}

\section{Disclosure of conflict of interest}

No conflict of interest. 


\section{Statement of ethical approval}

As the retrospective study data without any identifying patient information was taken, the ethics approval was waved off.

\section{Statement of informed consent}

Informed consent from the patient was taken to publish his case following with disclosing his personal information.

\section{References}

[1] Linhares I, Raposo T, Rodrigues A, Almeida A. Frequency and antimicrobial resistance patterns of bacteria implicated in community urinary tract infections: a ten-year surveillance study (2000-2009). BMC Infect Dis. 2013; 13: 19.

[2] Wagenlehner FM, Lichtenstern C, Rolfes C, et al. Diagnosis and management for urosepsis. Int J Urol. 2013; 20: 963-70.

[3] Martin GS, Mannino DM, Moss M. The effect of age on the development and outcome of adult sepsis. Crit Care Med. 2006; 34: 121.

[4] Tal S, Guller V, Levi S, et al. Profile and prognosis of febrile elderly patients with bacteremic urinary tract infection. J Infect. 2005; 50: 296305.

[5] Nitzan O, Elias M, Chazan B, Saliba W. Urinary tract infections in patients with type 2 diabetes mellitus: a review of prevalence, diagnosis, and management. Diabetes MetabSyndrObes Targets Ther. 2015; 8:129-36.

[6] Venmans LMAJ, Hak E, Gorter KJ, Rutten GEHM. Incidence and antibiotic prescription rates for common infections in patients with diabetes in primary care over the years 1995 to 2003. Int J Infect Dis. 2009; 13(6): e344-51.

[7] Ansari S, Nepal HP, Gautam R, Shrestha S, Neopane P, Gurung G, et al. Community-acquired multi-drug resistant clinical isolates of Escherichia coli.

[8] Kumarasamy KK, Toleman MA, Walsh TR, Bagaria J, Butt F, Balakrishnan R, et al. Emergence of a new antibiotic resistance mechanism in India, Pakistan, and the UK: a molecular, biological, and epidemiological study. Lancet Infect Dis. 2010; 10(9): 597-602.

[9] World health organisation. Antimicrobial resistance.

[10] Trivedi M, Patel V, Soman R, Rodriguez C, Singhal T. The outcome of treating ESBL infections with carbapenems vs. non-carbapenem antimicrobials. J Assoc Physicians India. 2012; 60: 28-30. 